\title{
Retrospective study of prognostic value of DNA ploidy and proliferative activity in neuroblastoma
}

\author{
S N Huddart, K R Muir, S E Parkes, J R Mann, M C G Stevens, F Raafat, K Smith
}

\begin{abstract}
Aim-To assess the prognostic value of age and stage at diagnosis, site of primary tumour, cell ploidy and $\mathrm{N}-m y c$ copy number in children with neuroblastoma.

Methods-Flow cytometry was used to determine the cellular DNA content of paraffin wax embedded archival material from 69 cases of neuroblastoma and was successful in 52 .

Results-The age, stage, and survival distribution of the sampled cases was not significantly different from that in a larger population based series. There were seven diploid ("non-aneuploid") and 45 aneuploid (including two tetraploid and four triploid) tumours. The 10 year survival was significantly better for cases of aneuploid rather than diploid tumours $(p<0.05)$. An important new finding was that 10 year survival was also significantly better for tumours with a low percentage of cells in $S$ phase $(\mathrm{p}<0.03)$.

Conclusion-The percentage of cells in $S$ phase, a measure of the proliferative activity of the tumour, correlated with prognosis in neuroblastoma. This should be measured with other biological features of the disease, such as $\mathbf{N}$-myc copy number, when prognostic indicators are being assessed.
\end{abstract}

(F Clin Pathol 1993;46:1101-1104)

Neuroblastoma is the most common extracranial solid tumour in children. Survival figures remain poor, especially for older children with advanced disease. There is increasing interest in the biological behaviour and prognosis of neuroblastoma. Age and stage at diagnosis and site of primary tumour are established prognostic factors, ${ }^{1}$ and to these have been added cell ploidy ${ }^{2}$ and N-myc oncogene copy number, ${ }^{3}$ both of which have been suggested as having strong prognostic value. Most children with the tumour excrete catecholamine metabolites in their urine, so asymptomatic cases can be screened for. Such screening programmes are only likely to be successful if cases detected by urine testing have the same disease, biologically, as those who present clinically. Evidence is now accumulating to suggest that this may not be the case. A recent study by Kaneko et $\mathrm{al}^{4}$ showed that children detected by a screening pro- gramme were more likely to have near triploid (3n) or hypertetraploid tumours $(>4 n$ ) with a single copy of the N-myc oncogene; those presenting clinically were more likely to have diploid tumours with multiple copies of the $\mathrm{N}$-myc oncogene. Retrospective review of histopathological material has the advantage that relatively large numbers of patients can be analysed, their management will not have been influenced by knowledge of a prognostic factor measured some time after treatment, and follow up information will have already been collected.

Before establishing a pilot study for neuroblastoma screening in the West Midlands, we performed an analysis of the disease over the 32 years 1957-88. To assess, retrospectively, the prognostic importance of DNA variables in this population, we used flow cytometry on paraffin wax sections of tumour biopsy specimens taken at diagnosis. Hedley et $a l^{5}$ showed that flow cytometry could accurately be used to determine cellular DNA content on paraffin wax embedded pathological material. Look $e t a l^{2}$ described the use of flow cytometry to measure cellular DNA content in 35 tumours obtained at surgery. They concluded that diploidy was more common in tumours of advanced stage, whereas hyperdiploidy was associated with earlier disease stage and a better response to chemotherapy. Others have applied quantitative DNA analysis to neuroblastoma and have divided tumours into aneuploid and "nonaneuploid" (to emphasise the abnormal karyotype of those tumours with a near diploid DNA content) ${ }^{6}$

Pathological material taken at diagnosis had been stored over many years in The Children's Hospital, Birmingham thus enabling a retrospective review of prognostic factors to be done on a relatively large series with long follow up. The aim of this study was to use flow cytometry to analyse this archival material for cellular DNA content, allowing for a more rapid assessment of the prognostic importance of ploidy than could be gained from a prospective study.

\section{Methods}

Cases were identified from a 32 year retrospective study of neuroblastoma in the population defined by the boundaries of the West Midlands Health Authority. During this period, 239 cases were diagnosed in children under 15 years of age. Review and confirmation of the diagnosis was completed in over 
$95 \%$ of cases by a panel of paediatric histopathologists. Paraffin wax sections $(40 \mu \mathrm{m})$ suitable for flow cytometry were available for 69 of the children, most sections obtained being from children treated at The Children's Hospital. Material tended to be more easily available from tumours located in the thorax than in the adrenal gland $-19 \%$ of the population based series had their primary tumour in the thorax and $53 \%$ in the adrenal, whereas $25 \%$ of cases with material suitable for DNA analysis had thoracic primaries and $33 \%$ had adrenal tumours.

Paraffin wax embedded material was prepared using a modified version of that described by Hedley et al ${ }^{5}$ : $40 \mu \mathrm{m}$ sections were dewaxed with xylene and rehydrated through $100 \%, 90 \%$, and $70 \%$ alcohol to distilled water. Sections were digested for 60 minutes at $37^{\circ} \mathrm{C}$ in $1 \mathrm{ml} 1 \%$ pepsin (activity $2500-3000 \mathrm{U}$; Sigma) in 0.9\% sodium chloride ( $\mathrm{pH} \mathrm{1.5)} \mathrm{with} \mathrm{occasional} \mathrm{vortex} \mathrm{agita-}$ tion. Cell counts were performed at this stage to ensure that more than 10000 cells were present. The cell suspension was centrifuged at $2500 \mathrm{rpm}$ for 10 minutes and resuspended in $1 \mathrm{ml} 10 \mathrm{mM}$ TRIS ( $\mathrm{pH} 7 \cdot 0$ ) containing $5 \mu \mathrm{M} \mathrm{MgC}_{12}$, as described by Deitch et al. ${ }^{7}$ Specimens were filtered through a $100 \mu \mathrm{m}$ pore size nylon mesh and stored overnight at $4^{\circ} \mathrm{C}$. Immediately before analysis, specimens were stained with $0.5 \mathrm{ml}$ propidium iodide $(1 \mathrm{mg} / \mathrm{ml}$ in TRIS, $\mathrm{pH} 7.0)$ and $0.5 \mathrm{ml}$ ribonuclease $(2 \mathrm{mg} / \mathrm{ml}$ in TRIS, $\mathrm{pH} 7 \cdot 0)$ added to the $1 \mathrm{ml}$ suspension and incubated for 15 minutes at $37^{\circ} \mathrm{C}$. Cell clumping was reduced by gently passing the suspension through a 19 gauge needle several times, followed by filtration through a $35 \mu \mathrm{m}$ pore sized nylon gauze. The specimens were kept on ice until analysis on the same day.

Flow cytometry of at least 30000 cells was performed using a Becton-Dickinson FACS 440 cell sorter with an Argon Laser light source. Samples from normal tonsil served as controls. DNA histograms were accepted when the coefficient of variation (CV) of the $\mathrm{G}_{0} / \mathrm{G}_{1}$ peak was less than $10 \%$.

The proportion of cells in the synthetic (S), gap $2\left(\mathrm{G}_{2}\right)$, and mitotic $(\mathrm{M})$ phases of the cell cycle was used as an index of proliferative activity of the tumour. The $S$ phase fraction was calculated using $T-2 x(A+B)$ after Koss et al. ${ }^{8}$ The tumours were divided into high or low $S$ phase according to whether the percentage of cells in $S$ phase was more or less than the median value.

Patients' records were reviewed to determine Evans' stage, ${ }^{1}$ primary site of disease, and age at diagnosis. Treatment varied according to the protocols used at the time of diagnosis. Those children diagnosed towards the start of the series received vitamin $B_{12}$, whereas those diagnosed later received multiagent chemotherapy, such as vincristine, cyclophosphamide, and doxorubicin with or without DTIC or vincristine, cisplatin, teniposide and cyclophosphamide.

Overall survival for each ploidy group was estimated using the Kaplan-Meier method $^{9}$ with differences in survival assessed using the log-rank test. ${ }^{10}$

\section{Results}

Flow cytometry was performed on material from 69 cases and was successful in 52 $(74 \%)$. Histograms of the remaining 17 cases showed excessive cellular debris and a $G_{0} / G_{1}$ peak CV of greater than $10 \%$. Excluded cases had a median age at diagnosis of 33 months (40.5 months for successful cases). Eighty three percent of their tumours originated in the adrenal gland (compared with $40 \%$ for successful cases) and $62.5 \%$ of these cases were stage 4 at diagnosis (compared with $48 \%$ ). Of the 52 successful cases, $38 \%$ were from the first half of the study period $(1957-72)$ and $62 \%$ from the second (1973-88).

There were only seven diploid ("nonaneuploid") and 45 aneuploid (including two tetraploid and four triploid) tumours (figs 1 and 2). The median age of the children at diagnosis was 40.5 months (diploid 41 months, aneuploid 40 months; not significant). All diploid tumours were stage III or IV; only $75 \%$ of aneuploid tumours had advanced stage disease. For cases in which the site of the primary tumour was known, four of $5(80 \%)$ diploid tumours originated in the adrenal compared with 12 of $41(30 \%)$ of aneuploid tumours. The results are likely to

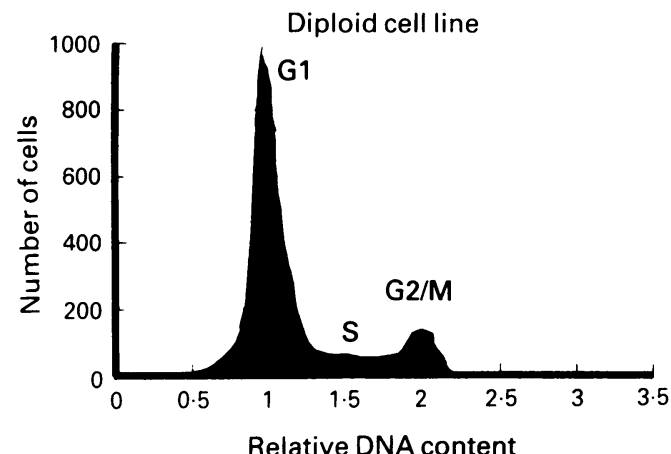

Figure 1 Histogram of diploid (non-aneuploid) tumour. The major peak represents cells in $G_{1}$ and the smaller second peak, cells in $G_{2}+M$ phase of the cell cycle. Those cells between these two peaks are in the $S$ (synthetic) phase of the cell cycle.

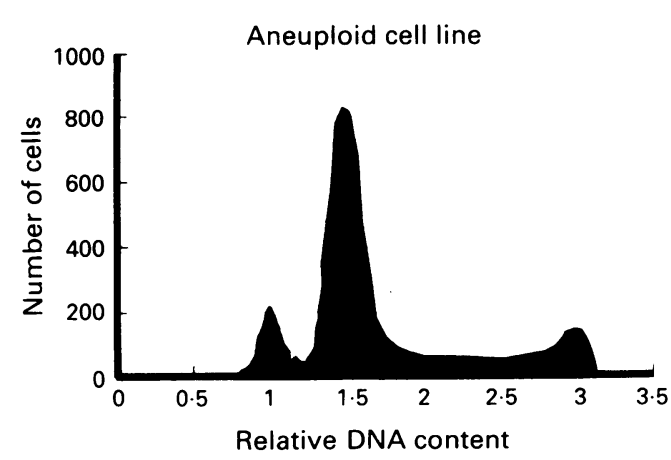

Figure 2 Histogram of an aneuploid tumour. The first peak represents normal diploid stromal cells with the second, larger peak representing the $G_{1}$ phase of the aneuploid cell line. 
Figure 3 Ten year survival curves showing a significant $(p=0.02)$ survival advantage for tumours with less than $14 \%$ of cells undergoing DNA synthesis.

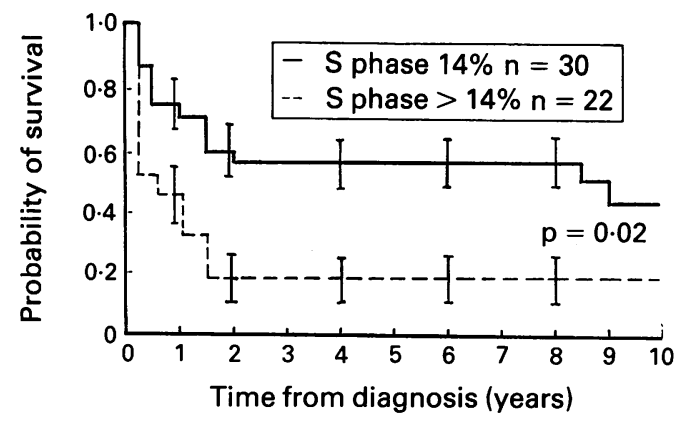

be biased towards easily resectable tumours (as these children will tend to have had an open biopsy and will have pathology slides remaining for analysis).

Cell proliferative activity, as reflected by the percentage of $S$ phase cells, was determined in 42 patients. The results varied from 3 to 38 per cent (median $14 \%$ ), with the tumours from 20 patients having $S$ phase below or equal to $14 \%$, and 22 tumours having $S$ phase above $14 \%$.

Ten year survival was significantly better for those 45 children with aneuploid tumours than for the seven children who had diploid tumours, although the number of cases in this particular analysis was obviously very small $(p<0.05)$. This agrees with the published work of Look et al. ${ }^{2}$ Those 20 children with tumours having a low percentage of cells in $S$ phase had a better 10 year prognosis, however, than the 22 children with tumours in S-phase above $14 \%(p=0.02)$ (fig 3$)$.

\section{Discussion}

Despite being the most common solid tumour in infancy, neuroblastoma is relatively rare (with an incidence of 7.2 cases per million children a year in the West Midlands. ${ }^{11}$ It is therefore essential to study retrospective data to accumulate information on sufficient cases for reliable analysis. We were able to measure DNA ploidy on archival material using flow cytometry and collated the results with survival data.

Age and stage of disease at diagnosis have long been important prognostic factors in neuroblastoma. More recently, additional biological markers have been used to assess prognosis and to plan treatment. These include: N-myc oncogene amplification, pattern of excretion of catecholamine metabolites, serum ferritin and serum neurone specific enolase and cell ploidy. The precise role and interplay of these factors remains to be established.

Using population based data on a larger series than previously used, we have confirmed the work of others ${ }^{2}$ that five year survival is significantly better for children with aneuploid tumours. Aneuploidy has been accepted as a marker of malignancy and neuroblastoma is unusual in that aneuploid tumours carry the more favourable prognosis compared with-for example, colonic carcinoma. ${ }^{12}$
The percentage of cells synthesising DNA in preparation for mitosis is an index of cellular activity. We have also shown that a higher percentage of cells in $S$ phase indicates a worse prognosis. This contrasts with findings reported for acute lymphoblastic leukaemia, in which a correlation was noted between a low percentage of S-phase cells and lack of initial response to induction treatment. ${ }^{13}$ This may reflect the lower number of target cells (those actively dividing) for the chemotherapy to act on. In neuroblastoma the biological mechanism may be different, however, and recent work by Eilers et $a l^{14}$ has shown that activation of the proto-oncogene MYC activates $a$-prothymosin which may act to elicit entry into and progression through the cell cycle. There may therefore be a link between amplified $\mathrm{N}-m y c$ and an increased percentage of cells in S-phase in neuroblastoma.

The results of this study confirm the use of cell ploidy as a prognostic factor in neuroblastoma, although the number of aneuploid cases is relatively small. This study has also identified the potential value of measuring the percentage of cells in the synthetic phase of the cell cycle. As screening for neuroblastoma becomes more widespread, the prognostic markers of cases detected clinically and by screening should be measured to determine whether the two populations are biologically the same. This study suggests that the proliferative index of neuroblastoma may be a useful prognostic marker in addition to those already in use and should be used to confirm the biological entity of tumours detected by screening.

Despite being accrued from a large population base and over a relatively long period of time, only 69 cases were potentially available for analysis. This reflects the limited availability of sufficient retrospective blocks from which a relatively thick $(40 \mu \mathrm{m})$ section is available. With successful analysis possible in 52 cases, this however, represents the largest series available to date. The cases in the sample were shown to be representative of the larger population based series.

These important results now need to be validated in further data sets, preferably collected prospectively, and including further information on treatment and other clinical and pathological factors, to assess their independent role in identifying prognosis.

We acknowledge the assistance of Roger Bird in the University of Birmingham Medical School for help with flow Cniversity of Birmingham Medical School for help with flow cytometr, Dr AH Cameron and Dr JR Pincott for the review of histopathological slides and Miss K Snow of the Children's Hospital for help with illustrations. This research has been supported by the Cancer Research Campaign and the Research Endowment Fund of the former United Birmingham Hospitals.

1 Evans AE, D'Angio GJ. A proposed staging for children with neuroblastoma Cancer 1971;27:374-8

2 Look AT, Hayes FA, Nitschke R, et al. Cellular DNA content as a predictor of response to chemotherapy in infants with unresectable neuroblastoma. $N$ Engl f $\mathrm{Med}$ 1984;311:231-5.

3 Brodeur GM, Seeger RC, Shwab M, Varmus HE, Bishop JM. Amplification of N-myc in untreated human neuroblastomas correlates with advanced disease stage. Science 1984;224:1121-4. 
4 Kaneko Y, Kanda N, Maseki N, et al. Current urinary mass screening for catecholamine metabolites at 6 months of age may be detecting only a small portion of high risk neuroblastomas: a chromosome and N-myc amplification study. $\mathcal{F}$ Clin Oncol 1990;8:2005-13.

5 Hedley DW, Friedlander ML, Taylor IW, Rugg CA, Musgrove EA. Method for analysis of cellular DNA content of paraffin-embedded pathological material using flow cytometry. $₹$ Histochem Cytochem 1983;31: using flow

6 Taylor SR, Locker J. A comparative analysis of nuclear DNA content and $\mathrm{N}$-myc gene amplification in neuroblastoma. Cancer 1990;65:1360-6.

7 Deitch AD, Law $H$, White RD. A stable propidium iodide procedure for flow cytometry. $\mathcal{F}$ Histochem Cytochem 1982;30:967-72.

8 Koss LG, Czerniak B, Herz F, et al. Flow cytometric measurements of DNA and other cell components in human tumors. Hum Pathol 1989;20:528-48.
9 Kaplan EL, Meier P. Non-parametric estimation from incomplete observations. $\mathcal{F}$ Am Stat Assoc 1958;53: 457-81.

10 Peto R, Peto J. Asymptotically efficient rank invariant test procedures. F $R$ Stat Soc $A$ 1972;135:185-216.

11 Huddart SN, Muir KR, Parkes SE, Mann JR, Stevens MCG, Raafat F. Neuroblastoma: A 32 year populationbased study-implications for screening. Med Pediatr Oncol 1993;21:96-102.

12 Jones DJ, Moore M, Schofield PF. Prognostic significance of DNA ploidy in colorectal cancer: A prospective flow cytometric study. Br f Surg 1988;75:28-33.

13 Look AT, Melvin SL, Williams DL, et al. Aneuploidy and percentage of S-phase cells determined by flow cytometry correlate with cell phenotype in childhood acute leukaemia. Blood 1982;60:959-67.

14 Eilers M, Schirm S, Bishop JM. The MYC protein activates transcription of the alpha-prothymosin gene. EMBO F 1991;10:133-41. 